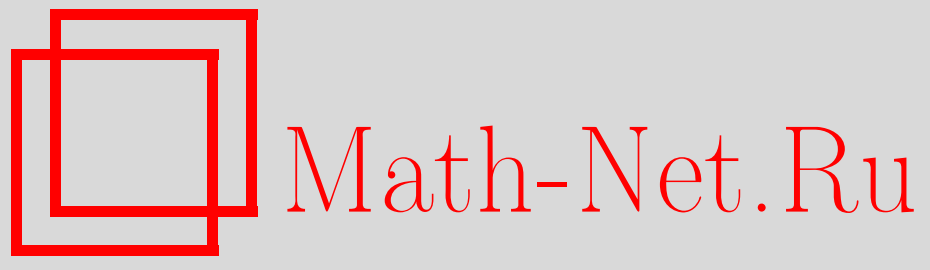

M. Beibel, H. R. Lerche, A note on optimal stopping of regular diffusions under random discounting, Теория вероятн. и ее примен., 2000, том 45, выпуск 4, 657-669

DOI: https://doi.org/10.4213/tvp497

Использование Общероссийского математического портала Math-Net.Ru подразумевает, что вы прочитали и согласны с пользовательским соглашением http: //www . mathnet.ru/rus/agreement

Параметры загрузки:

IP : 52.6 .47 .48

26 апреля 2023 г., 15:04:21

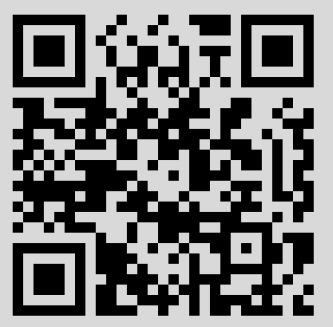


(C) $2000 \mathrm{r}$.

\author{
BEIBEL M.* , LERCHE H. R.*
}

\title{
A NOTE ON OPTIMAL STOPPING OF REGULAR DIFFUSIONS UNDER RANDOM DISCOUNTING
}

\begin{abstract}
Пусть $X$ - одномерная диффузия, удовлетворяюшая некоторым условиям регулярности, $A-$ положительный непрерывный аддитивный функционал от $X$ и $h$ - измеримая вещественнозначная функция. Предложен метод нахождения правила остановки $T^{*}$, которое максимизирует $\mathbf{E}\left\{e^{-A_{T}} h\left(X_{T}\right) 1_{\{T<\infty\}}\right\}$ по всем моментам остановки $T$ процесса $X$. Обсуждается ряд примеров, некоторые из них из области финансовой математики.
\end{abstract}

Ключевые слова и фразы: диффузия, обобщенная задача о выборе стоянки, оптимальная остановка, случайные потери.

\section{Introduction}

Let $B$ denote standard Brownian motion and let $r$ be a non-negative measurable function on the real numbers $\mathbf{R}$. Let us consider the problem of maximizing the gain

$$
\mathbf{E}\left(\exp \left\{-\int_{0}^{T} r\left(B_{s}\right) d s\right\}\left(B_{T}^{+}\right)^{\alpha} 1_{\{T<\infty\}}\right)
$$

for some $\alpha>0$ over all stopping times $T$ of $B$. To find an optimal stopping time for this and related problems we extend the approach of Beibel and Lerche [1]. In the present case that means to represent the payoff $\exp \left\{-\int_{0}^{t} r\left(B_{s}\right) d s\right\}\left(B_{t}^{+}\right)^{\alpha}$ as

$$
\exp \left\{-\int_{0}^{t} r\left(B_{s}\right) d s\right\} \psi\left(B_{t}\right) \frac{\left(B_{t}^{+}\right)^{\alpha}}{\psi\left(B_{t}\right)}
$$

where $\psi$ is chosen such that $\exp \left\{-\int_{0}^{t} r\left(B_{s}\right) d s\right\} \psi\left(B_{t}\right)$ becomes a positive local martingale and $\left(x^{+}\right)^{\alpha} / \psi(x)$ is a function which assumes its maximum

*Institut für Mathematische Stochastik, Universität Freiburg, Eckerstr., 1, 79104 Freiburg, Germany; e-mail: beibel@stochastik.uni-freiburg.de; lerche@stochastik.unifreiburg.de 
over $\mathbf{R}$ at some point $x^{*}>0$. Then we obtain for all stopping times $T$ of $B$ that

$$
\begin{aligned}
& \mathbf{E}\left(\exp \left\{-\int_{0}^{T} r\left(B_{s}\right) d s\right\}\left(B_{T}^{+}\right)^{\alpha} 1_{\{T<\infty\}}\right) \\
& \quad \leqslant\left(\sup _{x \in \mathbf{R}} \frac{\left(x^{+}\right)^{\alpha}}{\psi(x)}\right) \mathbf{E}\left(\exp \left\{-\int_{0}^{T} r\left(B_{s}\right) d s\right\} \psi\left(B_{T}\right) 1_{\{T<\infty\}}\right) \\
& \quad \leqslant \psi(0) \frac{\left(x^{*}\right)^{\alpha}}{\psi\left(x^{*}\right)} .
\end{aligned}
$$

In order to prove the optimality of $T^{*}=\inf \left\{t \geqslant 0 \mid B_{t}=x^{*}\right\}$, it is left to show that

$$
\mathbf{E}\left(\exp \left\{-\int_{0}^{T^{*}} r\left(B_{s}\right) d s\right\} \psi\left(B_{T^{*}}\right) 1_{\left\{T^{*}<\infty\right\}}\right)=\psi(0) .
$$

To be more explicit, let us consider the case $r(x)=r 1_{[0, \infty)}(x)$ for some positive constant $r$. Let

$$
\psi(x)= \begin{cases}\frac{1}{2}(\exp (\sqrt{2 r} x)+\exp (-\sqrt{2 r} x)) & \text { for } x \geqslant 0 \\ 1 & \text { for } x<0\end{cases}
$$

Then it holds that $\sup _{x \in \mathbf{R}}\left[\left(x^{+}\right)^{\alpha} / \psi(x)\right]<\infty$ and the supremum is attained at the unique positive solution $x^{*}$ of the implicit equation

$$
\sqrt{2 r} x\left(e^{\sqrt{2 r} x}-e^{-\sqrt{2 r} x}\right)=\alpha\left(e^{\sqrt{2 r} x}+e^{-\sqrt{2 r} x}\right) .
$$

The generalized Itô formula (see [6, IV.45.2]) yields that

$$
\exp \left\{-r \int_{0}^{t} 1_{(0, \infty)}\left(B_{s}\right) d s\right\} \psi\left(B_{t}\right)
$$

is indeed a positive local martingale. Moreover $\psi$ is nondecreasing. Therefore the process $\exp \left\{-\int_{0}^{t} r\left(B_{s}\right) d s\right\} \psi\left(B_{t}\right)$ is bounded by $\psi\left(x^{*}\right)$ for $0 \leqslant t \leqslant T^{*}$. Since $\mathbf{P}\left\{T^{*}<\infty\right\}=1$, this yields (1). Thus $T^{*}$ is an optimal stopping rule.

The approach just presented can be applied to a general class of optimal stopping problems. Let $X$ denote a one-dimensional regular diffusion in the sense of Feller, Itô, and McKean on some probability space $(\Omega, \mathscr{F})$. «Regular» here means that $\mathbf{P}\left\{T_{y}<\infty \mid X_{0}=x\right\}>0$ holds for all $y \in I$ and $x \in \operatorname{int}(I)$, with $T_{y}=\inf \left\{t \geqslant 0 \mid X_{t}=y\right\}$. In the sequel we assume that the structure of $(\Omega, \mathscr{F})$ is such that there exist measurable shift operators $\theta_{s}$ for $s \geqslant 0$ with $X_{t+s}(\omega)=X_{t}\left(\theta_{s}(\omega)\right)$ for all $\omega \in \Omega$ and $s, t \geqslant 0$. Let $\mathscr{F}_{t}^{X}=\sigma\left(X_{s}, 0 \leqslant s \leqslant t\right)$. Let $h$ be a measurable function and $r$ a positive constant. Let $\left(A_{s} ; s \geqslant 0\right)$ be a non-negative continuous additive functional of $X$, which means that $A$ satisfies the following conditions:

i) $A_{t} \in[0, \infty)$ for $0 \leqslant t<\infty$;

ii) $A_{t}$ is measurable with respect to $\mathscr{F}_{t}^{X}$; 
iii) $A_{s+t}=A_{s}+A_{t} \circ \theta_{s}$;

iv) $A_{t}$ is continuous in $t$.

We will consider the following general optimal stopping problem.

Problem 1. Find a stopping time $T^{*}$ of $X$ that maximizes

$$
\mathbf{E}\left(e^{-A_{T}} h\left(X_{T}\right) 1_{\{T<\infty\}} \mid X_{0}=x\right)
$$

among all stopping times $T$ of $X$.

Note that the stopping time $T \equiv+\infty$ yields the expected payoff 0 . Hence we will never stop whenever $h\left(X_{t}\right)<0$. This means we can always replace $h$ by $\max \{h, 0\}$.

The case of deterministic discounting $A_{t}=r t$ is treated by Mucci [5] and Salminen [7]. Their methods and conditions on $h$ differ somewhat from ours. Under additional technical conditions on the drift and diffusion coefficient of $X$, Bensoussans and Lions [2] treat the case, where $h$ is bounded and $A_{t}=\int_{0}^{t} r\left(X_{s}\right) d s$ with a positive bounded measurable function $r$ with $r \geqslant \beta$ for some strictly positive constant $\beta$ (see $[2$, Theorem 3.19, p. 387]).

In the following the state space of $X$ is an interval $I$ on the real line. The interior of $I$ will be denoted by $\operatorname{int}(I)$. An endpoint $z$ of $I$ with $z \in I$ will be called closed endpoint. We will sometimes write $\mathbf{P}_{x}$ and $\mathbf{E}_{x}$ instead of $\mathbf{P}\left\{\cdot \mid X_{0}=x\right\}$ and $\mathbf{E}\left(\cdot \mid X_{0}=x\right)$ respectively. For later use we introduce the following classification of closed boundary points. A closed endpoint $z$ of $I$ is called a reflecting boundary point if for some $y \in \operatorname{int}(I)$ (and hence for all $y \in I$ ) it holds that $\mathbf{P}\left\{T_{y}<\infty \mid X_{0}=z\right\}>0$ and it is called an absorbing boundary point if $\mathbf{P}\left\{T_{y}<\infty \mid X_{0}=z\right\}=0$ holds for all $y \in \operatorname{int}(I)$.

This paper has the following structure. In Section 2 we use the approach of Itô and McKean [3, Section 4.6]) and construct suitable functions $\psi$ for which $e^{-A_{t}} \psi\left(X_{t}\right)$ are local martingales. Section 3 contains our main results. In Section 4 we present further examples. The proofs of the results in Section 3 are given in Section 5, and Section 6 covers briefly some extensions.

\section{The functions $\psi_{+}$and $\psi_{-}$}

In this section we follow the ideas of Section 4.6 in [3]. The proofs are similar to the arguments there and in Section V.46 of [6]. We therefore omit them. Let $x_{0}$ denote some point in $\operatorname{int}(I)$. We then define the functions $\psi_{+}$ and $\psi_{-}$on $\operatorname{int}(I)$ by (compare [3, p. 128-129, equations $2 \mathrm{a}$ ) and $2 \mathrm{~b}$ )])

$$
\psi_{+}(x)= \begin{cases}\mathbf{E}_{x}\left(e^{-A\left(T_{x_{0}}\right)} 1_{\left\{T_{x_{0}}<\infty\right\}}\right) & \text { for } x \leqslant x_{0}, \\ {\left[\mathbf{E}_{x_{0}}\left(e^{-A\left(T_{x}\right)} 1_{\left\{T_{x}<\infty\right\}}\right)\right]^{-1}} & \text { for } x \geqslant x_{0},\end{cases}
$$

and

$$
\psi_{-}(x)= \begin{cases}{\left[\mathbf{E}_{x_{0}}\left(e^{-A\left(T_{x}\right)} 1_{\left\{T_{x}<\infty\right\}}\right)\right]^{-1}} & \text { for } x \leqslant x_{0}, \\ \mathbf{E}_{x}\left(e^{-A\left(T_{x_{0}}\right)} 1_{\left\{T_{x_{0}}<\infty\right\}}\right) & \text { for } x \geqslant x_{0} .\end{cases}
$$


The values at closed endpoints of $I$ can be computed by using the continuity properties of $\psi_{+}$and $\psi_{-}$. Let

$$
M_{t}^{(+)}=e^{-A(t)} \psi_{+}\left(X_{t}\right) \quad \text { and } \quad M_{t}^{(-)}=e^{-A(t)} \psi_{-}\left(X_{t}\right) .
$$

Lemma 1. $M_{t}^{(+)}$is a uniformly integrable $\mathbf{P}_{x}$-martingale on $0 \leqslant t \leqslant T_{b}$ for all $b \geqslant x$ and $M_{t}^{(-)}$is a uniformly integrable $\mathbf{P}_{x}$-martingale on $0 \leqslant t \leqslant T_{a}$ for all $a \leqslant x$. For any $x \leqslant b$ we have

$$
\mathbf{E}_{x}\left(M_{T_{b}}^{(+)} 1_{\left\{T_{b}<\infty\right\}}\right)=\psi_{+}(x)
$$

and for any $x \geqslant a$ we have

$$
\mathbf{E}_{x}\left(M_{T_{a}}^{(-)} 1_{\left\{T_{a}<\infty\right\}}\right)=\psi_{-}(x) .
$$

Clearly $\psi_{+}(a)>0$ if $a$ is a reflecting left boundary point and $\psi_{+}(a)=0$ if $a$ is an absorbing left boundary point. Also $\psi_{-}(b)>0$ if $b$ is a reflecting right boundary point and $\psi_{-}(b)=0$ if $b$ is an absorbing right boundary point. Moreover $\psi_{+}(b)>0$ for any closed right endpoint $b$ and $\psi_{-}(a)>0$ for any closed left endpoint. Moreover:

Lemma 2. The functions $\psi_{+}$and $\psi_{-}$are continuous and strictly positive on $\operatorname{int}(I)$. If $a$ is a closed left endpoint of $I$, then

$$
\lim _{x \downarrow a} \psi_{+}(x)=\psi_{+}(a) \quad \text { and } \quad \lim _{x \downarrow a} \psi_{-}(x)=\psi_{-}(a) .
$$

If $b$ is a closed right endpoint of $I$, then

$$
\lim _{x \uparrow b} \psi_{+}(x)=\psi_{+}(b) \quad \text { and } \quad \lim _{x \uparrow b} \psi_{-}(x)=\psi_{-}(b) .
$$

The function $\psi_{+}$is nondecreasing on $I$ and $\psi_{-}$is nonincreasing on $I$.

When $A_{t}=\int_{0}^{t} r\left(X_{s}\right) d s$ is given with some non-negative measurable function $r$ on $I$, we may use the Feynman-Kac formula to determine the functions $\psi_{+}$and $\psi_{-}$(see [11, Section 12.3]). When $\mathscr{G}$ denotes the infinitesimal operator of $X$, then one has to solve the equation $\mathscr{G} \psi(x)=r(x) \psi(x)$ subject to the appropriate boundary conditions.

\section{Optimal stopping}

Let us first assume that for all $x \in I$ holds $\psi_{+}(x)>0$ and $\psi_{-}(x)>0$. This means that we have no absorbing boundary points. We will extend our results to absorbing boundaries in Section 6 . Theorem 1 below corresponds to the case, where the optimal payoff is infinite. Theorem 2 essentially covers the case, where the optimal stopping rule is «one-sided» whereas Theorem 3 treats the «two-sided» case.

We fix the starting point $X_{0}$ of the process $X$ at some point $x_{0} \in I$. Without loss of generality we may assume that the functions $\psi_{+}$and $\psi_{-}$are 
standardized in such a way that $\psi_{+}\left(x_{0}\right)=\psi_{-}\left(x_{0}\right)=1$. We may write the payoff as

$$
e^{-A_{t}} h\left(X_{t}\right)=e^{-A_{t}}\left(p \psi_{+}\left(X_{t}\right)+(1-p) \psi_{-}\left(X_{t}\right)\right) \frac{h\left(X_{t}\right)}{p \psi_{+}\left(X_{t}\right)+(1-p) \psi_{-}\left(X_{t}\right)}
$$

for any $p \in[0,1]$ and $0 \leqslant t<\infty$. The process $e^{-A_{t}}\left(p \psi_{+}\left(X_{t}\right)+(1-p) \psi_{-}\left(X_{t}\right)\right)$ is a positive local martingale and hence a supermartingale. This yields for any stopping time $T$ of $X$

$$
\mathbf{E}_{x_{0}}\left(e^{-A_{T}}\left(p \psi_{+}\left(X_{T}\right)+(1-p) \psi_{-}\left(X_{T}\right)\right) 1_{\{T<\infty\}}\right) \leqslant 1 .
$$

The problem of maximizing

$$
\mathbf{E}_{x_{0}}\left(e^{-A_{T}} h\left(X_{T}\right) 1_{\{T<\infty\}}\right)
$$

over all stopping times $T$ is equivalent to the problem of maximizing

$$
\frac{h(x)}{p \psi_{+}(x)+(1-p) \psi_{-}(x)}
$$

over all $x \in I$ for a proper choice of $p$. We have to consider five different cases. It is easy to see that our cases cover all possible functions $h$. However, they are not exclusive.

i) $\sup _{x \geqslant x_{0}, x \in I}\left[h(x) / \psi_{+}(x)\right]=+\infty$. Then $p=1$ is a proper choice and Theorem 1 applies.

ii) $\sup _{x \leqslant x_{0}, x \in I}\left[h(x) / \psi_{-}(x)\right]=+\infty$. Then $p=0$ is a proper choice and Theorem 1 applies.

iii) $0<C^{*}=\sup _{x \in I}\left[h(x) / \psi_{+}(x)\right]=\sup _{x \geqslant x_{0}, x \in I}\left[h(x) / \psi_{+}(x)\right]<\infty$. Then $p=1$ is a proper choice and Theorem 2 i) applies.

iv) $0<C^{*}=\sup _{x \in I}\left[h(x) / \psi_{-}(x)\right]=\sup _{x \leqslant x_{0}, x \in I}\left[h(x) / \psi_{-}(x)\right]<\infty$. Then $p=0$ is a proper choice and Theorem 2 ii) applies.

v) $0<\sup _{x \geqslant x_{0}, x \in I}\left(h(x) / \psi_{+}(x)\right)<\infty$ and $0<\sup _{x \leqslant x_{0}, x \in I} \frac{h(x)}{\psi_{-}(x)}<\infty$, and at the same time

$$
\sup _{x \leqslant x_{0}, x \notin I} \frac{h(x)}{\psi_{+}(x)}>\sup _{x \geqslant x_{0}, x \in I} \frac{h(x)}{\psi_{+}(x)} \text { and } \sup _{x \geqslant x_{0}, x \in I} \frac{h(x)}{\psi_{-}(x)}>\sup _{x \leqslant x_{0}, x \in I} \frac{h(x)}{\psi_{-}(x)} .
$$

Similar arguments as in [1] then provide the existence of a $p^{*} \in(0,1)$ such that

$$
\begin{aligned}
& \sup _{x \geqslant x_{0}, x \in I} \frac{h(x)}{p^{*} \psi_{+}(x)+\left(1-p^{*}\right) \psi_{-}(x)} \\
& \quad=\sup _{x \leqslant x_{0}, x \in I} \frac{h(x)}{p^{*} \psi_{+}(x)+\left(1-p^{*}\right) \psi_{-}(x)} .
\end{aligned}
$$

Now $p=p^{*}$ is a proper choice and Theorem 3 applies.

The following elementary example illustrates all possibilities. Let $X$ denote the three-dimensional Bessel process and put $X_{0}=1$. We have 
for $x, y \in(0, \infty)$ that $\mathbf{P}_{x}\left\{T_{y}<\infty\right\}=\min \{(y / x), 1\}$. Let $A \equiv 0$. Then $\psi_{+}(x)=1$ and $\psi_{-}(x)=1 / x$.

The cases i) and iii) are trivial.

Case ii). If $h_{2}(x)=1 / x^{2}$, then $\sup _{0<x \leqslant 1}\left[h_{2}(x) / \psi_{-}(x)\right]=+\infty$.

Case iv). If $h_{4}(x)=(1-x)^{+}$, then $\sup _{x>0}\left[h_{4}(x) / \psi_{-}(x)\right]=$ $\sup _{0<x \leqslant 1}\left[h_{4}(x) / \psi_{-}(x)\right]=\frac{1}{4}$. The supremum is assumed at $x=\frac{1}{2}$.

Case v). If

$$
h_{5}(x)=\left\{\begin{array}{cl}
1-x^{2} & \text { for } 0<x \leqslant 1 \\
\frac{1}{x}-\frac{1}{x^{3}} & \text { for } 1<x<\infty
\end{array}\right.
$$

then

$$
0<\sup _{x \geqslant 1} \frac{h_{5}(x)}{\psi_{+}(x)}=\frac{2}{3 \sqrt{3}}=\sup _{0<x \leqslant 1} \frac{h_{5}(x)}{\psi_{-}(x)}
$$

and at the same time

$$
\sup _{0<x \leqslant 1} \frac{h_{5}(x)}{\psi_{+}(x)}=1=\sup _{x \geqslant 1} \frac{h_{5}(x)}{\psi_{-}(x)} .
$$

Moreover $x h_{5}(x) /[1+x]=x(x-1)$ for $x \in(0,1]$ and $x h_{5}(x) /[1+x]=$ $(1 / x)(1-1 / x)$ for $x \in(1, \infty)$. Therefore

$$
\sup _{x \geqslant 1} \frac{h_{5}(x)}{0.5 \psi_{+}(x)+0.5 \psi_{-}(x)}=0.5=\sup _{0<x \leqslant 1} \frac{h_{5}(x)}{0.5 \psi_{+}(x)+0.5 \psi_{-}(x)} .
$$

So, $p^{*}=0.5$. Note that the supremum is attained at $x=0.5$ and $x=2$ respectively.

Theorem 1. If

$$
\sup _{x \geqslant x_{0}, x \in I} \frac{h(x)}{\psi_{+}(x)}=+\infty \quad \text { or } \sup _{x \leqslant x_{0}, x \in I} \frac{h(x)}{\psi_{-}(x)}=+\infty,
$$

then

$$
\sup _{T} \mathbf{E}_{x_{0}}\left(e^{-A_{T}} h\left(X_{T}\right) 1_{\{T<\infty\}}\right)=+\infty .
$$

Theorem 2. i) If

$$
0<C^{*}=\sup _{x \in I} \frac{h(x)}{\psi_{+}(x)}=\sup _{x \geqslant x_{0}, x \in I} \frac{h(x)}{\psi_{+}(x)}<\infty
$$

then

$$
\sup _{T} \mathbf{E}_{x_{0}}\left\{e^{-r T} h\left(X_{T}\right) 1_{\{T<\infty\}}\right\}=C^{*} .
$$

If there exists a point $x^{*} \geqslant x_{0}$ with $C^{*}=h\left(x^{*}\right) / \psi_{+}\left(x^{*}\right)$, then the supremum in (8) is attained for $T^{*}$ with $T^{*}=\inf \left\{t \geqslant 0 \mid X_{t}=x^{*}\right\}$.

ii) If

$$
0<C^{*}=\sup _{x \in I} \frac{h(x)}{\psi_{-}(x)}=\sup _{x \leqslant x_{0}, x \in I} \frac{h(x)}{\psi_{-}(x)}<\infty,
$$


then

$$
\sup _{T} \mathbf{E}_{x_{0}}\left\{e^{-\tau T} h\left(X_{T}\right) 1_{\{T<\infty\}}\right\}=C^{*}
$$

If there exists a point $x^{*} \leqslant x_{0}$ with $C^{*}=h\left(x^{*}\right) / \psi_{-}\left(x^{*}\right)$, then the supremum in (9) is attained for $T^{*}$ with $T^{*}=\inf \left\{t \geqslant 0 \mid X_{t}=x^{*}\right\}$.

Theorem 3. Let $p^{*}$ be such that

$$
\begin{aligned}
0 & <\sup _{x \geqslant x_{0}, x \in I} \frac{h(x)}{p^{*} \psi_{+}(x)+\left(1-p^{*}\right) \psi_{-}(x)} \\
& =\sup _{x \leqslant x_{0}, x \in I} \frac{h(x)}{p^{*} \psi_{+}(x)+\left(1-p^{*}\right) \psi_{-}(x)}=C^{*}<\infty,
\end{aligned}
$$

then

$$
\sup _{T}\left\{\mathbf{E}_{x_{0}} e^{-r T} h\left(X_{T}\right) 1_{\{T<\infty\}}\right\}=C^{*}
$$

If there exist points $x_{1}>x_{0}$ and $x_{2}<x_{0}$ such that

$$
\frac{h\left(x_{1}\right)}{p^{*} \psi_{+}\left(x_{1}\right)+\left(1-p^{*}\right) \psi_{-}\left(x_{1}\right)}=\frac{h\left(x_{2}\right)}{p^{*} \psi_{+}\left(x_{2}\right)+\left(1-p^{*}\right) \psi_{-}\left(x_{2}\right)}=C^{*},
$$

then the supremum in (10) is attained for $T^{*}=\inf \left\{t>0 \mid X_{t}=x_{1}\right.$ or $\left.X_{t}=x_{2}\right\}$.

\section{Further examples}

Let $B$ denote standard Brownian motion throughout this section.

4.1. Standard Brownian motion - state-dependent discounting. Put $X=B$ and $x_{0}=0$. Let $r(\cdot)$ be a non-negative measurable function on $\mathbf{R}$. The function $\phi(x, y)=\mathbf{E}_{x} \exp \left\{-\int_{0}^{T_{y}} r\left(X_{s}\right) d s\right\}$ is closely related to the Feynman-Kac functional $\exp \left(-\int_{0}^{t} r\left(X_{s}\right) d s\right)$ and the stationary Schrödinger equation $\frac{1}{2} \psi^{\prime \prime}=r \psi$.

a) We have already discussed the particular example $r(x)=r 1_{[0, \infty)}(x)$ for some positive constant $r$. The solution we have presented in the introduction can be viewed as a special case of our general theorems.

b) Let $r(x)=r x^{2}$ for some constant $r>0$. Put

$$
\psi(x)=e^{-x^{2} / 4} \frac{2^{5 / 4}}{\Gamma(1 / 2)} \int_{0}^{+\infty} e^{x t-t^{2} / 2} \frac{1}{\sqrt{t}} d t .
$$

In the notation of $[4$, p. $91-94] \psi(x)$ equals $2^{5 / 4} D_{-1 / 2}(-x)$; the function $D_{-1 / 2}$ is usually called Weber function (see also $[8$, Section 3$]$ ). The function $\psi$ solves $\psi^{\prime \prime}=\frac{1}{4} x^{2} \psi$ and we have $\psi(0)=1$. Hence $\psi(\sqrt{8 / r} x)$ is a solution of $\frac{1}{2} \psi^{\prime \prime}=r x^{2} \psi$. We have $\psi \geqslant 0$ and $\lim _{x \rightarrow-\infty} \psi(x)=0$. This yields that $\psi$ is bounded on the interval $(-\infty, y]$ for any $x \in \mathbf{R}$. 
If we apply the optional stopping theorem to the local martingale $\exp \left\{-r \int_{0}^{t} X_{s}^{2} d s\right\} \psi\left(\sqrt{8 / r} X_{t}\right)$, we obtain for $y \geqslant 0$

$$
\begin{aligned}
& \mathbf{E}_{0} \exp \left\{-r \int_{0}^{T_{y}} X_{s}^{2} d s\right\}=\frac{1}{\psi(\sqrt{8 / r} y)} \text { and } \\
& \mathbf{E}_{x} \exp \left\{-r \int_{0}^{T_{0}} X_{s}^{2} d s\right\}=\psi\left(\sqrt{\frac{8}{r}} x\right)
\end{aligned}
$$

for $x \leqslant 0$. According to (4) this provides $\psi_{+}(x)=\psi\left((8 / r)^{1 / 2} x\right)$. Suppose now we are interested in maximizing

$$
\mathbf{E}_{0}\left(\exp \left\{-r \int_{0}^{T} X_{s}^{2} d s\right\}\left(X_{T}^{+}\right)^{\alpha} 1_{\{T<\infty\}}\right)
$$

for some $\alpha>0$. We have $\lim _{x \rightarrow+\infty}\left[\sqrt{x} \psi(x) / e^{x^{2} / 4}\right]=K$ for some strictly positive constant $K$. This provides $\sup _{x \in \mathbf{R}}\left[\left(x^{+}\right)^{\alpha} / \psi_{+}(x)\right]=$ $\sup _{x \geqslant 0}\left[\left(x^{+}\right)^{\alpha} / \psi_{+}(x)\right]<\infty$. Theorem 2 i) yields that $T^{*}=\inf \{t \geqslant 0 \mid$ $\left.X_{t}=x^{*}\right\}$, where $x^{*}$ is the positive solution of the transcendental equation $\alpha \psi_{+}(x)=x \psi_{+}^{\prime}(x)$.

4.2. Standard Brownian motion - discounting with local time. Put $X=B$ and $x_{0}=0$. Let $L$ denote the local time of $B$ at zero. We will now discuss the problem of maximizing $\mathbf{E}_{0}\left(e^{-r L_{T}}\left(X_{T}^{+}\right)^{\alpha} 1_{\{T<\infty\}}\right)$ for some $\alpha, r>0$. Let

$$
\psi(x)= \begin{cases}1+r x & \text { for } x \geqslant 0 \\ 1 & \text { for } x<0\end{cases}
$$

The generalized Itô formula (see [6, IV.45.2]) yields $d\left[e^{-r L_{t}} \psi\left(X_{t}\right)\right]=$ $r e^{-r L_{t}} d X_{t}$. Therefore $e^{-r L_{t}}\left(1+r X_{t}^{+}\right)$is a positive local martingale. We have $\left|e^{-r L_{t}}\left(1+r X_{t}^{+}\right)\right| \leqslant 1+r x$ on $0 \leqslant t \leqslant T_{x}$ for any $x \geqslant 0$ and hence we obtain $\mathbf{E}_{0} \exp \left\{-r L_{T_{x}}\right\}=1 /(1+r x)$ for $x \geqslant 0$. For $x<0$ it holds that $\mathbf{P}_{x}\left\{L_{T_{0}}=0\right\}=1$. This yields together $\psi_{+}(x)=\psi(x)$.

a) If $\alpha>1$, we obtain $\sup _{x \geqslant 0}\left[\left(x^{+}\right)^{\alpha} / \psi_{+}(x)\right]=+\infty$, so Theorem 1 yields

$$
\sup _{T} \mathbf{E}_{0}\left(e^{-r L_{T}}\left(X_{T}^{+}\right)^{\alpha} 1_{\{T<\infty\}}\right)=+\infty .
$$

b) If $\alpha=1$, we obtain $\sup _{x \geqslant 0}\left[\left(x^{+}\right)^{\alpha} / \psi_{+}(x)\right]=1 / r$ and therefore

$$
\sup _{T} \mathbf{E}_{0}\left(e^{-r L_{T}}\left(X_{T}^{+}\right)^{\alpha} 1_{\{T<\infty\}}\right)=\frac{1}{r} .
$$

The function $x /(1+r x)$ does not attain its supremum over $[0,+\infty)$ at some finite point and so there exists no optimal stopping rule.

c) If $0<\alpha<1$, we obtain

$$
\sup _{x \geqslant 0} \frac{\left(x^{+}\right)^{\alpha}}{\psi_{+}(x)}=(1-\alpha)\left(\frac{\alpha}{1-\alpha}\right)^{\alpha}\left(\frac{1}{r}\right)^{\alpha}=C^{*}
$$


and this supremum is attained at $x^{*}=\alpha /[r(1-\alpha)]$. Theorem $\left.2 \mathrm{i}\right)$ implies

$$
\sup _{T} \mathbf{E}_{0}\left(e^{-r L_{T}}\left(X_{T}^{+}\right)^{\alpha} 1_{\{T<\infty\}}\right)=C^{*}=\mathbf{E}_{0}\left(e^{-r L_{T^{*}}}\left(X_{T^{*}}^{+}\right)^{\alpha} 1_{\left\{T^{*}<\infty\right\}}\right),
$$

where $T^{*}=\inf \left\{t>0 \mid X_{t}=\alpha /[(1-\alpha) r]\right\}$.

4.3. Russian options. Let $\mu \in \mathbf{R}$ and $\sigma>0$. Let

$$
S_{t}=\exp \left\{\sigma B_{t}+\left(\mu-\frac{\sigma^{2}}{2}\right) t\right\}
$$

and $M_{t}=\max _{0 \leqslant s \leqslant t} S_{s}$. Then $d S_{t}=\mu S_{t} d t+\sigma S_{t} d B_{t}$. Let $r>\mu$. We now consider the problem of maximizing $\mathbf{E}\left(e^{-r T} M_{T} 1_{\{T<\infty\}}\right)$ over all stopping times $T$ of $S$. Shepp and Shiryaev [9] proved the following result.

Let

$\gamma_{1,2}=-\left(\frac{\mu}{\sigma^{2}}-\frac{1}{2}\right) \mp \sqrt{\frac{2 r}{\sigma^{2}}+\left(\frac{\mu}{\sigma^{2}}-\frac{1}{2}\right)^{2}} \quad$ and $\quad \alpha=\left(\frac{1-1 / \gamma_{1}}{1-1 / \gamma_{2}}\right)^{1 /\left(\gamma_{2}-\gamma_{1}\right)}$.

Then

$$
\sup _{T} \mathbf{E}\left(e^{-r T} M_{T}\right)=\mathbf{E}\left(e^{-\tau T^{*}} M_{T^{*}}\right)
$$

where $T^{*}=\inf \left\{t>0 \mid M_{t} / S_{t}=\alpha\right\}$.

To obtain this result we first follow the approach of Shepp and Shiryaev [10] and rewrite the problem appropriately. Let $X_{t}=M_{t} / S_{t}$. Let $\widetilde{\mathbf{P}}$ denote the probability measure given by

$$
\left.\frac{d \widetilde{\mathbf{P}}}{d \mathbf{P}}\right|_{\sigma\left(B_{s}, 0 \leqslant s \leqslant t\right)}=e^{\sigma B_{t}-\left(\sigma^{2} / 2\right) B_{t}}=e^{-\mu t} S_{t}
$$

Let $\tilde{\mathbf{E}}$ denote the expectation with respect to $\widetilde{\mathbf{P}}$. Then for all stopping times $T$ of $S$

$$
\begin{aligned}
\mathbf{E}\left(e^{-\tau T} M_{T} 1_{\{T<\infty\}}\right) & =\mathbf{E}\left(e^{-(r-\mu) T} e^{-\mu T} S_{T} X_{T} 1_{\{T<\infty\}}\right) \\
& =\widetilde{\mathbf{E}}\left(e^{-(r-\mu) T} X_{T} 1_{\{T<\infty\}}\right)
\end{aligned}
$$

The process $X$ is a regular diffusion under $\widetilde{\mathbf{P}}$ with state space $[1, \infty)$ and instantaneous reflection at 1 . The infinitesimal generator of $X$ in $(1, \infty)$ is equal to

$$
\frac{\sigma^{2}}{2} x^{2} \frac{d}{d x^{2}}-\mu x \frac{d}{d x} \text {. }
$$

This means that $X$ behaves like an exponential Brownian motion with drift $-\mu$, diffusion coefficient $\sigma$ and instantaneous reflection at 1 . To maximize $\widetilde{\mathbf{E}}\left(e^{-(r-\mu) T} X_{T} 1_{\{T<\infty\}}\right)$ over all stopping times $T$ of $X$, let

$$
H(x)=\frac{\eta_{2}}{\eta_{2}-\eta_{1}} x^{\eta_{1}}-\frac{\eta_{1}}{\eta_{2}-\eta_{1}} x^{\eta_{2}}
$$


Here $\eta_{1}<0$ and $\eta_{2}>1$ are the two roots of

$$
\frac{\sigma^{2}}{2} x^{2}-\left(\mu+\frac{\sigma^{2}}{2}\right) x=(r-\mu) \text {. }
$$

Note that $\eta_{1,2}=1-\gamma_{2,1}$. Then

$$
\frac{\sigma^{2}}{2} x^{2} H^{\prime \prime}(x)-\left(\mu+\frac{\sigma^{2}}{2}\right) H^{\prime}(x)=(r-\mu) H(x)
$$

and $H^{\prime}(1)=0$. Moreover $H(x) \geqslant 0$ for $x \geqslant 1$ and $H(1)=1$. So, $\exp \{-(r-$

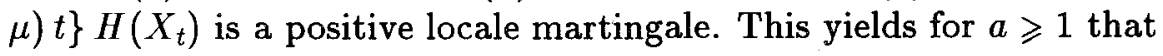

$$
\widetilde{\mathbf{E}}\left(e^{-(r-\mu) T_{a}} 1_{\left\{T_{a}<\infty\right\}}\right)=\widetilde{\mathbf{E}}\left(e^{-(r-\mu) T_{a}} H\left(X_{T_{a}}\right) \frac{1}{H\left(X_{T_{a}}\right)} 1_{\left\{T_{a}<\infty\right\}}\right)=\frac{1}{H(a)} .
$$

Note that $\widetilde{\mathbf{P}}\left\{T_{a}<\infty\right\}=1$ for all $a>1$ (see [10]) and $0 \leqslant H\left(X_{t}\right) \leqslant$ $\sup _{1 \leqslant x \leqslant a} H(x)<\infty$ for $0 \leqslant t \leqslant T_{a}$. The function $x / H(x)$ assumes its maximum over $[1, \infty)$ uniquely at $x^{*}=\alpha$. Therefore $T^{*}=T_{x^{*}}$. Theorem $2 \mathrm{i}$ ) now yields the assertion with $\psi_{+}(x)=H(x)$.

\section{Proofs}

P r o of of Theorem 1. Suppose $\sup _{x \geqslant x_{0}, x \in I}\left[h(x) / \psi_{+}(x)\right]=$ $+\infty$. Then there exists a sequence $x_{n} \geqslant x_{0}$ with $\lim _{n \rightarrow \infty}\left[h\left(x_{n}\right) / \psi_{+}\left(x_{n}\right)\right]=$ $+\infty$. Let $S_{n}=T_{x_{n}}$. Lemma 1 and (5) yield

$$
\mathbf{E}_{x_{0}}\left(e^{-A\left(S_{n}\right)} h\left(X_{S_{n}}\right) 1_{\left\{S_{n}<\infty\right\}}\right)=\frac{h\left(x_{n}\right)}{\psi_{+}\left(x_{n}\right)}
$$

Hence

$$
\sup _{T} \mathbf{E}_{x_{0}}\left(e^{-A_{T}} h\left(X_{T}\right) 1_{\{T<\infty\}}\right) \geqslant \sup _{n} \frac{h\left(x_{n}\right)}{\psi_{+}\left(x_{n}\right)}=+\infty .
$$

A similar argument applies to the case $\sup _{x \leqslant x_{0}, x \in I}\left[h(x) / \psi_{-}(x)\right]=+\infty$.

$\mathrm{Pr}$ o of of $\mathrm{T}$ he or e m $2 \mathrm{i}$ ). There exists a sequence $x_{n} \geqslant x_{0}$ with

$$
\lim _{n \rightarrow \infty} \frac{h\left(x_{n}\right)}{\psi_{+}\left(x_{n}\right)}=\sup _{x \in I} \frac{h(x)}{\psi_{+}(x)} .
$$

Let $S_{n}=T_{x_{n}}$. Lemma 1 and (5) yield

$$
\mathbf{E}_{x_{0}}\left(e^{-A\left(S_{n}\right)} h\left(X_{S_{n}}\right) 1_{\left\{S_{n}<\infty\right\}}\right)=\frac{h\left(x_{n}\right)}{\psi_{+}\left(x_{n}\right)} .
$$

Hence

$$
\sup _{T} \mathbf{E}_{x_{0}}\left(e^{-A_{T}} h\left(X_{T}\right) 1_{\{T<\infty\}}\right) \geqslant \sup _{n} \frac{h\left(x_{n}\right)}{\psi_{+}\left(x_{n}\right)}=\sup _{x \in I} \frac{h(x)}{\psi_{+}(x)} .
$$

On the other hand (6) yields for any stopping time $T$ of $X$ that

$$
\mathbf{E}_{x_{0}}\left(e^{-A_{T}} h\left(X_{T}\right) 1_{\{T<\infty\}}\right)=\mathbf{E}_{x_{0}}\left(M_{T}^{(+)} \frac{h\left(X_{T}\right)}{\psi_{+}\left(X_{T}\right)} 1_{\{T<\infty\}}\right) \leqslant \sup _{x \in I} \frac{h(x)}{\psi_{+}(x)} .
$$


Now suppose $C^{*}=h\left(x^{*}\right) / \psi_{+}\left(x^{*}\right)$ for some $x^{*} \geqslant x_{0}$. Lemma 1 yields for the stopping time $T^{*}=\inf \left\{t>0 \mid X_{t}=x^{*}\right\}$ that

$$
\mathbf{E}_{x_{0}}\left(e^{-r T^{*}} h\left(X_{T^{*}}\right) 1_{\left\{T^{*}<\infty\right\}}\right)=C^{*} \mathbf{E}_{x_{0}} M_{T^{*}}^{(+)}=C^{*} .
$$

The proof of Theorem 2 ii) is similar to the proof of Theorem $2 \mathrm{i}$ ).

$\mathrm{Pr}$ o of of $\mathrm{Th}$ eorem 3 . There exist sequences $x_{n}^{(+)} \geqslant x_{0}$ and $x_{n}^{(-)} \leqslant x_{0}$ such that

$$
\begin{aligned}
& \lim _{n \rightarrow \infty} \frac{h\left(x_{n}^{(+)}\right)}{p^{*} \psi_{+}\left(x_{n}^{(+)}\right)+\left(1-p^{*}\right) \psi_{-}\left(x_{n}^{(+)}\right)} \\
& =C^{*}=\lim _{n \rightarrow \infty} \frac{h\left(x_{n}^{(-)}\right)}{p^{*} \psi_{+}\left(x_{n}^{(-)}\right)+\left(1-p^{*}\right) \psi_{-}\left(x_{n}^{(-)}\right)} .
\end{aligned}
$$

Let $S_{n}$ denote the stopping time $S_{n}=\inf \left\{t \geqslant 0 \mid X_{t}=x_{n}^{(+)}\right.$or $\left.X_{t}=x_{n}^{(-)}\right\}$. Obviously we have $S_{n} \leqslant \inf \left\{t \geqslant 0 \mid X_{t}=x_{n}^{(+)}\right\}$and $S_{n} \leqslant \inf \left\{t \geqslant 0 \mid X_{t}=\right.$ $\left.x_{n}^{(-)}\right\}$and so Lemma 1 yields $\mathbf{E}_{x_{0}}\left[\left(p^{*} M_{S_{n}}^{(+)}+\left(1-p^{*}\right) M_{S_{n}}^{(-)}\right) 1_{\left\{S_{n}<\infty\right\}}\right]=1$. Hence

$$
\begin{array}{r}
\mathbf{E}_{x_{0}}\left(e^{-A\left(S_{n}\right)} h\left(X_{S_{n}}\right) 1_{\left\{S_{n}<\infty\right\}}\right) \\
\geqslant \min \left\{\frac{h\left(x_{n}^{(+)}\right)}{p^{*} \psi_{+}\left(x_{n}^{(+)}\right)+\left(1-p^{*}\right) \psi_{-}\left(x_{n}^{(+)}\right)},\right. \\
\left.\frac{h\left(x_{n}^{(-)}\right)}{p^{*} \psi_{+}\left(x_{n}^{(-)}\right)+\left(1-p^{*}\right) \psi_{-}\left(x_{n}^{(-)}\right)}\right\}
\end{array}
$$

and consequently $\sup _{T} \mathbf{E}_{x_{0}}\left(e^{-A(T)} h\left(X_{T}\right) 1_{\{T<\infty\}}\right) \geqslant C^{*}$. On the other hand (6) yields

$$
\sup _{T} \mathbf{E}_{x_{0}}\left(e^{-A(T)} h\left(X_{T}\right) 1_{\{T<\infty\}}\right) \leqslant \sup _{x \in I} \frac{h(x)}{p^{*} \psi_{+}(x)+\left(1-p^{*}\right) \psi_{-}(x)}=C^{*} .
$$

\section{Extensions}

6.1. Absorbing boundaries. We can also treat the case of absorbing boundaries. Let us suppose that $a$ is a left absorbing boundary point and that we have no right absorbing boundary point. The cases

- of a right absorbing boundary point but no left absorbing boundary point,

- of a right absorbing boundary point and a left absorbing boundary point

can be treated in a similar fashion. 
Now $\psi_{+}(a)=0$ and $\psi_{-}(x)>0$ for all $x \in I$. The case $x_{0}=a$ is trivial. Let $x_{0}>a$. The situation, where Theorem 2 ii) applies requires no changes. If $h(a)=0$, one simply has to replace $\sup _{x \leqslant x_{0}, x \in I}\left[h(x) / \psi_{+}(x)\right]$ and $\sup _{x \in I}\left[h(x) / \psi_{+}(x)\right]$ by $\sup _{a<x \leqslant x_{0}, x \in I}\left[h(x) / \psi_{+}(x)\right]$ and $\sup _{x \in I, x>a}\left[h(x) / \psi_{+}(x)\right]$ respectively. Then one can apply Theorem $2 \mathrm{i}$ ) and Theorem 3 . Therefore we assume

$$
\sup _{x \geqslant x_{0}, x \in I} \frac{h(x)}{\psi_{-}(x)}>\sup _{x \leqslant x_{0}, x \in I} \frac{h(x)}{\psi_{-}(x)}
$$

and $h(a)>0$.

We now have to look at two different cases separately. Let $\tilde{h}(x)=$ $h(x) 1_{\{x>a\}}$. It holds for any stopping time $T$ that

$$
\mathbf{E}_{x_{0}}\left(e^{-A_{T}} h\left(X_{T}\right) 1_{\{T<\infty\}}\right) \geqslant \mathbf{E}_{x_{0}}\left(e^{-A_{T}} \tilde{h}\left(X_{T}\right) 1_{\{T<\infty\}}\right)
$$

Theorem 1 now yields $\sup _{T} \mathbf{E}_{x_{0}}\left(e^{-A_{T}} h\left(X_{T}\right) 1_{\{T<\infty\}}\right)=+\infty$ if

$$
\sup _{x \geqslant x_{0}, x \in I} \frac{\widetilde{h}(x)}{\psi_{+}(x)}=\sup _{x \geqslant x_{0}, x \in I} \frac{h(x)}{\psi_{+}(x)}=+\infty .
$$

If $\sup _{x \geqslant x_{0}, x \in I}\left[h(x) / \psi_{+}(x)\right]<\infty$, we obtain together with (11) the existence of some $p^{*} \in(0,1)$ satisfying $(7)$. That means we are now in the situation covered by Theorem 3 . It is intuitively clear that Theorem 2 i) cannot apply if $h(a)>0$ since for any $x>x_{0}$ we have

$$
\mathbf{E}_{x_{0}}\left(e^{A\left(T_{x}\right)} h\left(X_{T_{x}}\right) 1_{\left\{T_{x}<\infty\right\}}\right)<\mathbf{E}_{x_{0}}\left(e^{A\left(T_{x} \wedge T_{a}\right)} h\left(X_{T_{x} \wedge T_{a}}\right) 1_{\left\{T_{x} \wedge T_{a}<\infty\right\}}\right) .
$$

6.2. Terminal times. We can also cover situations, where the process $X$ is eventually killed. Let $\xi$ be a terminal time of $X$; that is a stopping time of $X$ with the property that $\xi=s+\xi \circ \theta_{s}$ on the event $\{\xi>s\}$. After possibly switching to a smaller state space, we may assume that $\mathbf{P}\left\{T_{y}<\xi \mid X_{0}=x\right\}>0$ holds for all $y \in I$ and $x \in \operatorname{int}(I)$. Our method can be adapted to deal with the problem of finding a stopping time $T$ that maximizes

$$
\mathbf{E}\left(e^{-A(T)} h\left(X_{T}\right) 1_{\{T<\xi\}}\right) \text {. }
$$

In this case the functions $\psi_{+}$and $\psi_{-}$in (3) and (4) have to be replaced by

$$
\psi_{+}(x)= \begin{cases}\mathbf{E}_{x}\left(e^{-A\left(T_{x_{0}}\right)} 1_{\left\{T_{x_{0}}<\xi\right\}}\right) & \text { for } x \leqslant x_{0}, \\ {\left[\mathbf{E}_{x_{0}}\left(e^{-A\left(T_{x}\right)} 1_{\left\{T_{x}<\xi\right\}}\right)\right]^{-1}} & \text { for } x \geqslant x_{0},\end{cases}
$$

and

$$
\psi_{-}(x)= \begin{cases}\mathbf{E}_{x_{0}}\left[\left(e^{-A\left(T_{x}\right)} 1_{\left\{T_{x}<\xi\right\}}\right)\right]^{-1} & \text { for } x \leqslant x_{0} \\ \mathbf{E}_{x}\left(e^{-A\left(T_{x_{0}}\right)} 1_{\left\{T_{x_{0}}<\xi\right\}}\right) & \text { for } x \geqslant x_{0}\end{cases}
$$


6.3. Discounting with functionals which assume negative values. One might also think of applying our approach to problems, where $A$ assumes positive and negative values. For a particular example, where $A_{t}$ changes sign, see Section 2.6 in [1]. In this situation we can still follow the arguments in Section 2 and construct the functions $\psi_{+}$and $\psi_{-}$. Under suitable integrability conditions, like $\mathbf{E}_{x}\left(e^{-A\left(T_{y}\right)} 1_{\left\{T_{y}<\infty\right\}}\right)<\infty$ for all $x, y$ the processes $M_{t}^{-}$and $M_{t}^{+}$will become local martingales and Lemma 1 will hold. Then we are able to give analogues of Theorem 1 and Theorem 2 i) and ii). Unfortunately Lemma 2 will in general fail to hold and so we are not able to prove the existence of a $p^{*}$ satisfying (7). Therefore we can not give an analogue of Theorem 3 in this case.

Acknowledgments. A preliminary version of this manuscript only dealt with deterministic discounting. We thank Prof. Heinrich v. Weizsäcker for valuable discussions, in which he raised the question of path-dependent discounting.

\section{REFERENCES}

1. Beibel M., Lerche H.R. A new look at optimal stopping problems related to Mathematical Finance. -- Statist. Sinica, 1997, v. 7, p. 93-108.

2. Bensoussan A., Lions J.-L. Applications of Variational Inequalities in Stochastic Control. Amsterdam: North-Holland, 1982, 564 p.

3. Ito K., McKean H. P. Diffusion Processes and their Sample Paths. Berlin: SpringerVerlag, 1965.

4. Magnus W., Oberhettinger $F$. Formulas and Theorems for the Functions of Mathematical Physics. New York: Chelsea, 1954.

5. Mucci A.G. Existence and explicit determination of optimal stopping times. Stochastic Process. Appl., 1978, v. 8, p. 33-58.

6. Rogers L.C.G., Williams D. Diffusions, Markov Processes, and Martingales. V. 2: Itô's Calculus. Chichester: Wiley, 1987, 475 p.

7. Salminen $P$. Optimal stopping of one-dimensional diffusions. - Math. Nachr., 1985, v. 124 , p. $85-101$.

8. Shepp L. A. On the integral of the absolute value of the pinned Wiener process. Ann. Probab., 1982, v. 10, p. 234-239.

9. Shepp L.A., Shiryaev A.N. The Russian option: reduced regret. - Ann. Appl. Probab., 1993, v. 3, p. 631-640.

10. Шелn Л. А., ІІиряев A.H. Новый взгляд на расчеты «Русского опциона». Теория вероятн. и ее примен., 1994, т. 39, в. 1, с. 130-149.

11. Weizsäcker $H$. von, Winkler G. Stochastic Integrals. Braunschweig: Vieweg, 1990, $332 \mathrm{p}$. 